\title{
Study of Plasma Detachment in a Simplified 2D Geometry using UEDGE
}

\author{
M. Groth, A.M. Mahdavi, G.D. Porter, T.D. Rognlien
}

This article was submitted to

The $8^{\text {th }}$ International Workshop on Plasma Edge Theory in Fusion Devices, Espoo, Finland, September 10-12, 2001

Lawrence

Livermore

National

Laboratory

\section{August 31, 2001}

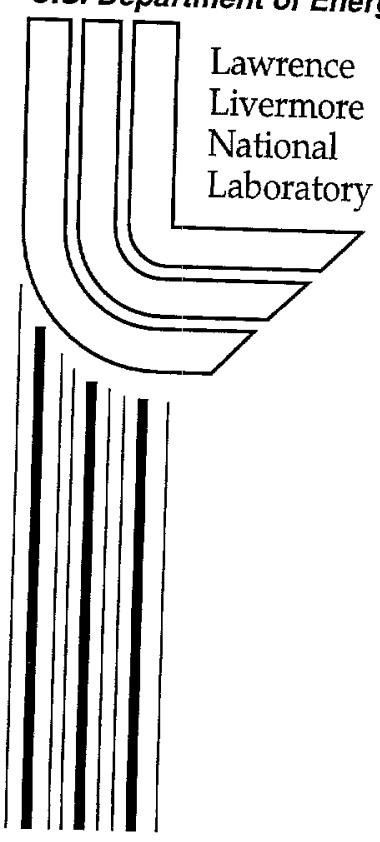




\section{DISCLAIMER}

This document was prepared as an account of work sponsored by an agency of the United States Government. Neither the United States Government nor the University of California nor any of their employees, makes any warranty, express or implied, or assumes any legal liability or responsibility for the accuracy, completeness, or usefulness of any information, apparatus, product, or process disclosed, or represents that its use would not infringe privately owned rights. Reference herein to any specific commercial product, process, or service by trade name, trademark, manufacturer, or otherwise, does not necessarily constitute or imply its endorsement, recommendation, or favoring by the United States Government or the University of California. The views and opinions of authors expressed herein do not necessarily state or reflect those of the United States Government or the University of California, and shall not be used for advertising or product endorsement purposes.

This is a preprint of a paper intended for publication in a journal or proceedings. Since changes may be made before publication, this preprint is made available with the understanding that it will not be cited or reproduced without the permission of the author.

This report has been reproduced directly from the best available copy.

Available to DOE and DOE contractors from the Office of Scientific and Technical Information P.O. Box 62, Oak Ridge, TN 37831

Prices available from (423) 576-8401 http://apollo.osti.gov/bridge/

Available to the public from the National Technical Information Service

U.S. Department of Commerce 5285 Port Royal Rd., Springfield, VA 22161 http://www.ntis.gov/

OR

Lawrence Livermore National Laboratory

Technical Information Department's Digital Library

http://www.llnl.gov/tid/Library.html 
Contrib. Plasma Phys. vol (year) num, $\mathrm{p}-\mathrm{p}$

\title{
Study of Plasma Detachment in a Simplified 2D Ge- ometry using UEDGE
}

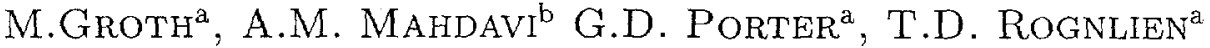 \\ ${ }^{a}$ LaWrence Livermore National Laboratory, Livermore, CA \\ 94551, USA

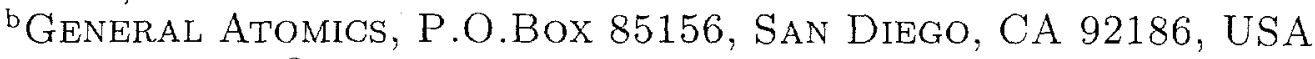 \\ E-MAIL:GROTH@FUSION.GAT.COM
}

\begin{abstract}
The location of the ionisation front in a generic, detached plasma has been studied in a two-dimensional slab geometry by varying the core plasma density and input heating power. The ratio of the recombination to ionisation current and the momentum losses in the computational domain have been used to quantify the degree of detachment. Contours of constant ratio of these parameter in the core plasma density - heating power paramter space show that at high input power higher momentum losses can be achieved even at low density. High fraction of recombination, however, require high core densities to sufficiently separate the plasma from the target plate.
\end{abstract}

\section{Introduction}

The detachment of the scrape-off layer (SOL) plasma in tokamaks is one of the key provision in the design of future fusion devices, providing the means to reduce the particle and heat loads onto the material surface to tolerable levels [1]. A detached plasma is characterised by two main features that co-exist synergetically: a radiation enhanced region and a recombination-dominated zone, where the gross of the plasma heat and momentum are dissipated, respectively. Radiation losses reduce the plasma temperature to drop to sufficiently low temperatures, that allowing ion-neutral friction to adequately occur; in return, ion-neutral collisions slow down the plasma flow, thus giving the plasma sufficient time require to recombine. These processes, which generally occur in different regions of the SOL, require plasma temperatures below $10 \mathrm{eV}$. As a result, an ionisation front is formed which is well-separated from the target surfaces.

In a one-dimensional picture, the plasma temperature in the divertor SOL of tokamaks is determined by the influx of heat from the core plasma and the outflux of heat due to divertor plate heating and radiation. Neutral particles resulting from the recycling processes, whether volumetric or at the wall, provide the energy and momentum sink for the impinging plasma ions. In two dimensions, however, and depending on its rate, radial transport can be of great important, but is difficult to implement analytically. Numerical codes can aid our understanding of the interplay between the physics processes that are involved. Here, the numerical code package UEDGE [2] is used to simulate and examine plasma detachment of generic plasmas in a simplified two-dimensional (2D) geometry [3]. 


\section{UEDGE in $2 \mathrm{D}$ slab geometry}

The UEDGE code is a two-dimensional multi-fluid plasma code which can run with a variety of geometries [2]. Plasma transport along the magnetic field is assumed classical [4] with flux-limits imposed to simulate kinetic effects and prevent excessive parallel thermal transport in regions of steep temperature gradients. Perpendicular transport is assumed to be diffusive and anomalous, with spatially constant ion diffusion coefficient; here, $D_{\perp}=0.5 \mathrm{~m}^{2} / \mathrm{s}$, and thermal diffusivity, $\chi_{\perp}=0.7 \mathrm{~m}^{2} / \mathrm{s}$, for both electrons and ions. Neutrals arising from recycling at the target are treated as a fluid, with the neutral diffusivity determined by charge exchange (CX) and elastic scattering rates [3]. Volumetric momentum and energy losses are described by Braginskii's transport equation for the plasma momentuum and energy [4], which are solved by UEDGE in poloidal, $\mathrm{x}$-, and radial, $\mathrm{y}$-direction of the $2 \mathrm{D}$ geometry [3] in this work. Thereby, a plasma is assumed that extends $1 \mathrm{~m}$ in poloidal and $0.05 \mathrm{~m}$ in radial direction. The toroidal field is taken to be constant at $5 T$, and the poloidal field is constant at $0.3 T$, giving a field pitch angle of $3.43^{\circ}$.

The particle and energy flow into the SOL is set in UEDGE by the core plasma density, ncore, and particle diffusivity, and the total heating power, $P_{t o t}$, and the thermal diffusivity at the core boundary grid cells, respectively. Particles are removed from the system by means of ion pumping at the target plate (Recycling coefficient $R_{P}=0.98$ ) and neutral pumping at the outer and private plasma walls (Albedo $\alpha_{0}=0.99$ ). Zero temperature and particle flux gradients are assumed at the outer and private plasma walls. The boundary condition at the target, $x=1.0 \mathrm{~m}$, follows the Bohm criterion [5], i.e. $M_{i, x=1}=1$. The sheath heat transmission coefficients, $\gamma_{i}$ and $\gamma_{e}$, for the ions and electrons are 2.5 and 4.0 , respectively.

\section{Plasma detachment in UEDGE slab geometry: the base case solution}

In the base case, the core plasma density is set to ncore $=7.5 \cdot 10^{19} \mathrm{~m}^{-3}$. This gives, together with $D_{\perp}=0.5 \mathrm{~m}^{2} / \mathrm{s}$, a particle in flux of $4.1 \cdot 10^{20}$ particles per second. In steady-state, particles are removed at a rate of $4.0 \cdot 10^{20}$ per second as neutrals at the outer wall, and the remaining $1 \cdot 10^{19}$ particles per second as ions at the target plate. The input power of $1 \cdot 10^{4} \mathrm{~W}$ is exhausted as hydrogenic radiation that is associated with ionisation of recycling neutrals, and also recombination, $9.5 \cdot 10^{3} \mathrm{~W}$, while the divertor plate heating is $5 \cdot 10^{2} \mathrm{~W}$. The divertor plasma is detached and the ionisation front is approximately $0.09 \mathrm{~m}$ separated from the solid surface.

The SOL above the $\mathrm{x}$-point is characterised by a radial inflow of particles and heat from the core plasma. The corresponding poloidal variation of the significant terms in the momentum and electron energy equation is shown for the separatrix flux tube in Fig.1. The ion parallel velocity, expressed as the fraction of the ion sound speed (Mach number) and the electron temperature are given by the right-hand scale of the plots, respectively. The inflow of particles and heat leads to a linearly rising parallel particle and heat flow. Across the x-point, momentum and heat is lost due to radial momentum diffusion and power flow into the private flux region (PFR). These processes occur as the shear in parallel velocity between the core and SOL plasma are large at, and the radial temperature and density gradients are reversed below the $\mathrm{x}$-point. The plasma temperature and the poloidal momentum drop significantly around the $\mathrm{x}$-point, which is a $2 \mathrm{D}$ effect that would not occur in a $1 \mathrm{D}$ treatment. 

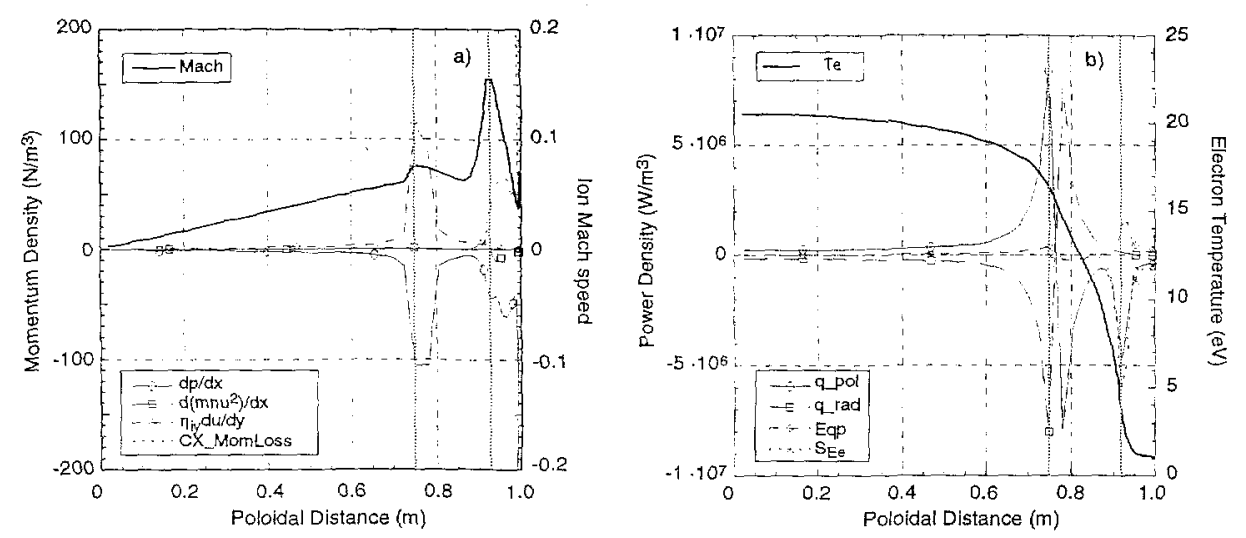

Fig. 1: Poloidal variation of the significant terms in the momentum (a) and energy equation (b) along the separatrix flux tube (base case). The ion speed (Mach number) and the electron temperature are given on the righthand $y$-axis. The approximate location of the $x$-point and the ionisation front are indicated by the gray, vertical lines.

The outflow of heat into the PFR causes the plasma temperature to drop to a point where heat losses due to ionisation become significant, thus the plasma temperature is reduced even faster. In the UEDGE cases shown here, the temperature, at which radiation is the dominant term is of the order $5-10 \mathrm{eV}$, since only hydrogenic radiation losses are considered. This region is defined as the ionisation-dominated region (IDR). The radiated power is supported by a combination of parallel energy flow from the upstream SOL, and heating from the ions due to ion- electron temperature equipartition. As the radial temperature gradient relaxes within the IDR, the radial heat losses into the PFR are insignificant. In order to sustain heat conduction, the parallel temperature gradient steepens rapidly between 5 and $10 \mathrm{eV}$, until, at $5 \mathrm{eV}$, parallel heat convection becomes the major heat transport process.

The plasma that stretches from the IDR to the target is cold $(\sim 1 \mathrm{eV})$ and dense $\left(\sim 10^{20} \mathrm{~m}^{-3}\right)$, and therefore dominated by recombination. We defined this region as the recombination-dominated region (RDR). The ion velocity is at its maximum at the entrance to the RDR, $M=0.15$. Due to CX collisions occuring within the RDR, this velocity decreases significantly. As UEDGE has to converge with an ion speed equivalent to $M=1$ at the target, the Mach number increases rapidly in the grid cells that are directly adjacent to the plate.

\section{Effect of core density and input power on detached plasma}

Experimentally, the core plasma density and heating power are the principal, external input parameter; their influence on the conditions of the detached plasma is therefore of great importance.

At fixed core plasma density, the total input power across the core boundary is varied, at fixed thermal diffusivity, from $5 \cdot 10^{3} \mathrm{~W}-2 \cdot 10^{4} \mathrm{~W}$. Increasing the input power raises the radial heat flux into the SOL, heats the electrons and ions in the upstream SOL, and concomitantly generates a larger heat flux in the parallel field direction. Below the $\mathrm{x}$-point, the temperature gradient between the SOL and the 

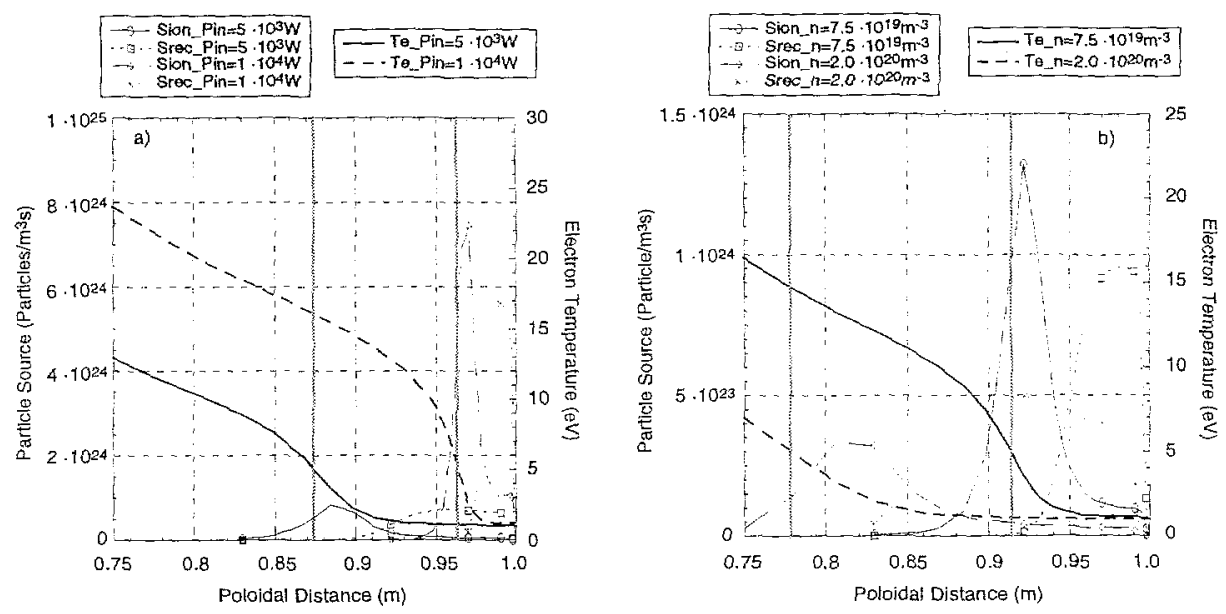

Fig. 2: Poloidal variation of the ionisation and recombination sources along the separatrix flux tube: a) with increasing power, and b) increasing density. The changes in the poloidal temperature profile due to the increase in power and density are shown on the right-hand $y$-axis. The gray, vertical lines indicate the approximate position of the ionisation front.

PFR steepens, and more momentum and heat is transported into the PFR. Still, the parallel heat fluxes that enter the IDR are larger, while the neutral density adjacent to the target increases. Facing this larger influx of heat from the upstream SOL and an enhanced plasma cooling downstream, the plasma temperature gradient within the IDR steepens dramatically, subsequently narrowing this region. As shown in Fig.2a, the ionisation front is forced to move closer to the target, narrowing the RDR, and the fraction of plasma recombining to neutrals ionising decreases. The CX momentum losses, integrated over the SOL and PFR, are smaller at high input power, with similar Mach numbers at the RDR exit.

Increasing the core plasma density at fixed heating power moves the ionisation front further away from the target plate (Fig.2b). In this study, the core density is varied from $5 \cdot 10^{18} \mathrm{~m}^{-3}$ to $2.5 \cdot 10^{20} \mathrm{~m}^{-3}$, while keeping the diffusion coefficient fixed. Raising the core plasma density cools the SOL plasma and lowers the heat fluxes along the field lines. At the highest core plasma density, the SOL plasma becomes as cold as $10 \mathrm{eV}$, so that heat convection becomes the dominant heat transport mechanism. The increased influx of particles into the divertor is followed by an increase in neutral density at the target plate; still, the temperature gradient within the IDR relaxes. The fraction of recombination to ionisation increases with increasing density. Increasing the neutral density at the target, by decreasing the neutral removal rate at the outer wall, moves the ionisation front further off the target and increases the ratio of recombination to ionisation. CX momentum losses are greater at high core plasma density. We conclude that the location of the ionisation front depends on both the upstream, core plasma conditions and re-ionisation processes that take place adjacent to the target plate.

Instead of attempting to approximate the momentum and energy equation in the RDR to derive an analytic expression for the location of the ionisation front, the approach taken here is to describe divertor detachment in the parameter space of core density and input power. For that purpose, the recombination to ionisation current 
ratio, $I_{r e c} / I_{i o n}$, and the CX momentum losses, $S_{m o m}$, are calculated to measure the degree of detachment. The former quantity is obtained by integrating over the PFR and divertor SOL domain,

$$
\frac{I_{\text {rec }}}{I_{\text {ion }}} \propto \frac{\left(n_{i}^{2}\langle\sigma v\rangle_{r e c}\right)_{R D R}}{\left(n_{0} n_{i}\langle\sigma v\rangle_{i o n}\right)_{I D R}}
$$

where $n_{i}$ and $n_{0}$ denote the ion and neutral density, and $\langle\sigma v\rangle_{\text {rec }}$ and $\langle\sigma v\rangle_{i o n}$ the recombination and ionisation rates in the RDR and IDR, respectively. CX momentum losses are determined by the drop in (static, $p$, and dynamic, $m_{i} n_{i} u_{i}^{2}$ ) pressure in parallel field direction,

$$
S_{m o m} \propto 1-\frac{\operatorname{mom}_{R D R}}{m_{\text {omp }}} \equiv 1-\frac{\left(p_{i}+p_{e}+m_{i} n_{i} u_{i}^{2}\right)_{R D R}}{\left(p_{i}+p_{e}\right)_{u p}}
$$

where $m_{i}$ is the ion mass. Momentum balance for attached cases can only be achieved when intergrating radially over the $\mathrm{PFR}$ and the SOL domain due to the radial outflow of momentum around the x-point. Upstream from the x-point, the pressure is integrated over the SOL domain only. The core plasma density input to UEDGE is normalised to the upstream density, $n_{u p}$, as given by the 2-Point Model [7],

$$
n_{u p-2 P M}=\sqrt{2 \frac{m_{i}}{e} q_{\|}^{2} \frac{\left(\frac{7}{2} \frac{q_{\|} L}{\kappa_{0 e}}\right)^{-4 / 7}}{\gamma^{2} e^{2} T_{t}}}
$$

where $e$ is the electron charge, $L$ is the length of the field line, $\gamma=6.5$, is the total sheath heat transmission coefficient, and, $\kappa_{0 e} \approx 2000$, is the Spitzer conductivity for single ion species of charge $Z_{i}$. A target plasma temperature, $T_{t}$, of $1 \mathrm{eV}$ is assumed, a temperature at which the divertor plasma is still in the attached, high recycling regime. The parallel heat flux density, $q_{\|}$, as in Eq.3, assumes that heat is transported by conduction only. This term is approximated by the input power, $P_{\text {in }}$, and the radial power scale length, $\lambda_{q \|}$, obtained by UEDGE,

$$
q_{\|}=\frac{P_{i n}}{\lambda_{q_{\|}}} \frac{B_{T}}{B_{P}}
$$

Typically, $\lambda_{q_{\|}}$varies between $5-10 \mathrm{~mm}$, giving a parallel heat flux density of $10^{7} \mathrm{~W} / \mathrm{m}^{2}$.

Resulting contours of constant $I_{r e c} / I_{i o n}$ and $p_{R D R} / p_{u p}$ ratio in the core densityheating power parameter space are shown Fig. $3 \mathrm{a}$ and $3 \mathrm{~b}$. At low density, the divertor plasma is dominated by ionisation, with negligible recombination; the ratio of $I_{\text {rec }} / I_{\text {ion }}$ is therefore low. To achieve significant recombination, the core plasma density must exceed about 3 at low input power, and about 2 at high power. The ratio of recombination to ionisation depends on the divertor plasma and neutral densitiy, but also whether the plasma flow is sufficiently low to permit significant recombination [8]. The competition between the time required for sufficient recombination and the ion transit time across the RDR is proportional to the inversed product of $n_{R D R} \Delta x, \Delta x$ being the width of the RDR. Increasing the core plasma density lowers the divertor density, but widens the width of the RDR. The opposite effect occurs when increasing the heating power. 

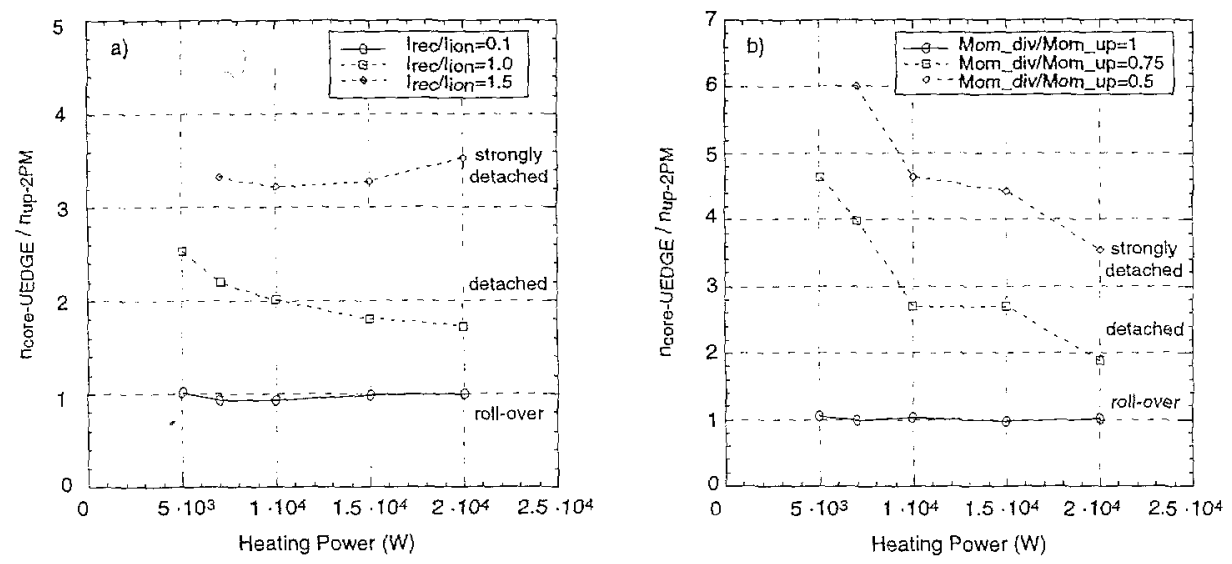

Fig. 3: Contours of constant recombination to ionisation (a) and momentum loss (b) in (normalised) core density - heating power space.

The CX momentum losses depend mainly on plasma and neutral density in the divertor, but also over which distance the ion-neutral friction acts, $S_{\text {mom }} \propto n_{0} n_{i} \Delta x$. Under detached conditions, increasing the core plasma density reduces the divertor plasma density, but raises neutral density and expands the RDR. When increasing the power both the plasma and neutral density is raised, but the width of the RDR becomes significantly shorter.

There is a trade-off between these processes, and depending on which process exerts the stronger influence, the recombination-to-ionisation ratio and the momentum losses increase or decrease. Operation at high density and high power seems to increase both the recombination-to-ionisation ratio and the CX momentum losses.

\section{Conclusions}

The location of the ionisation front in a generic plasma has been studied in a $2 \mathrm{D}$ geometry using UEDGE. On the high-temperature side, the location of the ionisation front is very sensitive to the particle and heat influx from the core into the divertor, but also the radial losses into the PFR. On the low temperature side, the critical parameters are radiation losses due to ionisation, CX momentum losses and the level of neutral density adjacent to the target. The location of the ionisation front has been examined as a function of the core plasma density and heating power. Increasing the core plasma density or decreasing the input power moves the ionisation front away from the target. Concomitantly, the region that is dominated by recombination expands, allowing more CX momentum loss collision to occur. Higher influxes of heat and larger neutral densities at high power lead to steep temperature gradients the region of high ionisation.

The dependence of the degree of detachment on core density and input power has been discussed in their parameter space. Based on the ratio of recombination to ionisation and downstream to upstream momentum, it has been shown that a higher degree of detachment can be achieved at the highest core density and heating input power. 


\section{Acknowledgments}

The authors thank Peter Stangeby of Toronto University, Institute of Aerospace Studies, for initiating this work. This work was performed of the US DoE by the University of California, LLNL under contract no. W-7405-ENG-48.

\section{References}

[1] Janeschitz, G. et al., J. Nucl. Mat. 220-222 (1995) 73

[2] RognLien, T.D. et al., J. Nucl. Mat. 196-198 (1992) 347

[3] Rognlien, T.D. et al., Contrib. Plasma. Phys. 38 (1998) 154

[4] Braginskit, S.I., in Reviews of Plasma Physics, edt. by Leontovich, M.A. (Consultants Bureau, New York, 1965) 1 (1965) 205

[5] Bohm, D., The Characteristics of Electrical Discharges in Magnetic FIELDS, (New York, 1949)

[6] Spitzer, L. And Harm, R., Phys. Rev. 89 (1958) 977

[7] Stangeby, P.C., The Plasma Boundary of Magnetic Fusion Devices, (Bristol, $2000), 227$

[8] Isler, R.C. et al., Phys. Plasmas 4 (1997) 2989

Received August 30, 2001. 